\title{
Análise dos ativos fiscais diferidos nas empresas do setor elétrico brasileiro
}

Analysis of deferred tax assets in companies of the Brazilian electric sector

Análisis de los activos fiscales diferidos en las empresas del sector eléctrico brasileño

\section{Maria Elisabeth Moreira Carvalho Andrade}

Doutora em Controladoria e Contabilidade pela Universidade de São Paulo (USP)

Professora do Programa de Pós-Graduação em Ciências Contábeis (UFU)

Endereço: Av. João Naves de Ávila, no 2121 - Bloco F - Sala 1F205 - Campus Santa Mônica

CEP: 38.400-902 - Uberlândia/MG - Brasil

E-mail: maria.elisabeth@ufu.br

Telefone: +55 (34) 3239-4176

Artigo recebido em 11/08/2017. Revisado por pares em 08/12/2019. Reformulado em 19/03/2020. Recomendado para publicação em 20/03/2019 por Carlos Eduardo Facin Lavarda (Editor-Chefe). Publicado em 30/03/2020. 


\section{Resumo}

O objetivo geral deste artigo foi analisar se o reconhecimento do ativo fiscal diferido (AFD) interfere na situação financeira das empresas do setor elétrico brasileiro, nas demonstrações societárias e nas demonstrações regulatórias no período de 2014 e 2015 . Foi utilizado o modelo Kronbauer et al. (2012a) para uma amostra de 30 empresas, foi demonstrado que há diferença significativa entre os índices Liquidez Geral e Endividamento quando do reconhecimento ou não do AFD, nos dados societários e regulatórios. Entretanto, nos dados societários para ambos os índices melhoram o desempenho financeiro da empresa quando há o reconhecimento do AFD. Com os dados regulatórios essa melhora ocorre com o índice de Liquidez Geral. Quanto ao endividamento o efeito é contrário, o reconhecimento do AFD aumenta o índice de endividamento, nas demonstrações regulatórias.

Palavras-chave: Ativos fiscais diferidos; Setor elétrico; Desempenho financeiro

\section{Abstract}

The general objective of this article was to analyze whether the recognition of deferred tax assets (DTA) interferes in the financial situation of companies in the Brazilian electricity sector, in the corporate and regulatory balance sheets in the period of 2014 and 2015. Was used the model of Kronbauer et al. (2012a) for a sample of 30 companies, it was shown that there is a significant difference between the General Liquidity and Indebtedness indexes when recognizing the DTA, in corporate and regulatory data. However, in the corporate data for both indexes improves the financial performance of the company when there is recognition of the DTA. With the regulatory data this improvement occurs with the General Liquidity index. As to indebtedness, the effect is contrary, the recognition of the DTA increases the indebtedness index in the regulatory statements. Keywords: Deferred tax assets; Electric sector; Financial performance

\section{Resumen}

El objetivo general de este artículo fue analizar si el reconocimiento del activo fiscal diferido (AFD) interfiere en la situación financiera de las empresas del sector eléctrico brasileño, en las demostraciones societarias y en las demostraciones regulatorias en el período de 2014 y 2015. Se utilizó el modelo Kronbauer et al. (2012a) para una muestra de 30 empresas, se mostró que hay diferencia significativa entre los índices Liquidez General y Endeudamiento en el reconocimiento o no del AFD, en los datos societarios y regulatorios. Sin embargo, en los datos societarios para ambos índices mejoran el desempeño financiero de la empresa cuando hay el reconocimiento del AFD. Con los datos regulatorios esta mejora ocurre con el índice de Liquidez General. En cuanto al endeudamiento el efecto es contrario, el reconocimiento del AFD aumenta el índice de endeudamiento, en los estados regulatorios.

Palabras clave: Activos fiscales diferidos; Sector eléctrico; Desempeño financiero

\section{Introdução}

A contabilidade brasileira sempre foi influenciada pela legislação fiscal. Com a adoção das normas internacionais de contabilidade a partir de 2008, uma das principais expectativas foi a distinção entre a contabilidade societária e contabilidade fiscal. Houve uma mudança de paradigma na contabilidade brasileira com o objetivo de evidenciar as demonstrações contábeis sob o enfoque econômico, ou seja, a essência sobre a forma.

Devido a esse novo prisma que com certeza repercute nos resultados das organizações e portanto, traz efeitos tributários presentes (ativos e passivos fiscais correntes) e futuros (ativos e passivos fiscais diferidos) foi emitido o normativo "Tributos sobre o Lucro" (CPC 32) com o objetivo de estabelecer o tratamento contábil dos efeitos fiscais atuais e futuros. Importante ressaltar que a Comissão de Valores Mobiliários (CVM) desde 1998 já havia deliberado sobre o 
tema (DELIBERAÇÃO No 273).

Entretanto, o CPC 32 propicia na decisão dos gestores de reconhecer o montante dos tributos diferidos, podendo ser conservador ou não, como por exemplo, melhorar a rentabilidade (MADEIRA; COSTA JÚNIOR, 2016).

O foco deste estudo são os ativos fiscais diferidos (AFDs) que são reconhecidos quando há uma diferença temporária entre a contabilidade societária e fiscal, como por exemplo, provisões para garantia. Entretanto, é a probabilidade de sua ocorrência que determinará se haverá o reconhecimento do ativo fiscal diferido.

Diante do exposto, levanta-se o seguinte problema de pesquisa, existe associação entre o índice de ativo fiscal diferido (IAFD) e as variáveis inerentes à situação financeira e econômica das empresas do setor elétrico brasileiro?

O objetivo geral deste artigo é averiguar se existe associação entre o índice de AFD - IAFD e as variáveis inerentes à situação financeira e econômica das empresas analisadas e discutir as diferenças entre a contabilidade societária e regulatória.

A escolha do setor elétrico justifica-se porque é um setor altamente regulamentado pela Agência Nacional de Energia Elétrica - ANEEL, sendo importante para o desenvolvimento econômico e social do país e as demonstrações financeiras mais próximas da realidade contribui para a tomada de decisão dos investidores e reguladores.

Este artigo está composto de mais quatro seções, além desta introdução. A próxima seção apresenta uma visão teórica sobre o tema abordado. A terceira traz os procedimentos metodológicos utilizados nessa pesquisa. A quarta e quinta seções são destinadas a apresentação e análise dos resultados e das considerações finais da pesquisa, respectivamente.

\section{Referencial Teórico}

\subsection{Evidenciação dos Impostos Diferidos e comportamento oportunista}

Ativos Fiscais Diferidos surgem de algumas diferenças temporárias quando se inclui receita ou despesa no resultado contábil em um período, e no resultado tributável em período diferente (PEREZ JUNIOR; OLIVERIA, 2010). Ainda segundo esses autores são exemplos de transações que resultam em AFD: provisões para garantias; provisões para riscos fiscais e outros litígios; valor contábil de ativo ajustado ao valor de mercado ou recuperável, compensação de prejuízos fiscais, entre outros.

Segundo Wasserman (2004, p.12) o AFD "representa ou deveria representar o benefício fiscal futuro a ser auferido quando essas despesas passarem a ser dedutíveis na apuração do lucro tributável".

$\mathrm{O}$ ativo fiscal diferido deve ser reconhecido se houver evidências do grau de certeza e possível futuro lucro tributável com o qual o ativo possa ser compensado (MARTINS; GELBCKE; SANTOS; IUDÍCIBUS, 2013, p. 397).

Madeira e Costa Júnior (2016) evidenciaram que os ativos fiscais diferidos são, em sua maioria, advindos de diferenças temporárias, apesar de haver um crescimento dos créditos fiscais referentes a prejuízos fiscais. O período do estudo foi de 2010 a 2013 em 68 empresas brasileiras não financeiras.

Ajustes temporários como a permissão da dedutibilidade de uma despesa ou a exigência de tributação de uma receita geram reflexos tributários (PAULO; MARTINS; CORRAR, 2007).

Esse reconhecimento pode influenciar no desempenho financeiro das empresas como mencionado nos estudos citados. Tal fato pode contribuir para comportamentos oportunistas, como gerenciamento de resultados (KRONBAUER; SOUZA; WEBBER; OTT, 2012a).

Na seção 2.4 são apresentados alguns estudos sobre o tema. Enfim, a não evidenciação dos AFD pode proporcionar comportamento oportunista. 
Segundo Paulo, Martins e Corrar (2007) quando as normas contábeis possibilitam diversos critérios na contabilização abrem caminho para os gestores optarem por métodos legais que possam impactar o desempenho da empresa.

O estudo de Herbon, Tutticci e Khor (2010) encontrou fortes evidências de que há gerenciamento nas demonstrações contábeis sobre o montante de ativos fiscais diferidos com o objetivo de alcançar as metas de lucro.

Rezende e Nakao (2012) encontraram evidências de que as medidas de gerenciamento de resultados são influenciadas por interesses econômicos relacionados à tributação.

Entretanto, ainda não há resultados robustos quanto ao tema, por isso mais pesquisas são necessárias.

\subsection{Diferenças entre a Contabilidade Societária e Regulatória do setor elétrico}

As concessionárias do setor elétrico estão sujeitas às normas contábeis emanadas de dois organismos: (i) o Comitê de Pronunciamentos Contábeis (CPC); e, (ii) a Agência Nacional de Energia Elétrica (ANEEL). Segundo Suzart, Souza, Carvalho, Riva, Martins e Salotti (2013) os dois órgãos buscam informações relevantes para usuários distintos. Enquanto as normas do CPC foca os investidores, as da ANEEL buscam prestar contas à sociedade sobre a gestão dos bens públicos e para a formação da tarifa cobrada pelo fornecimento de energia elétrica.

Os normativos que atualmente regulam o setor é a ICPC 01 (R1) - Contratos de Concessão emitida em 2009; ICPC 17 - Contratos de Concessão: Evidenciação emitida em 2011 e OCPC 05 - Contratos de Concessão emitida em 2010. E pela ANEEL, em 2010 foi editada a Resolução n. ${ }^{\circ}$ 396, que instituiu a contabilidade regulatória e atualizou o manual de contabilidade, denominado Manual de Contabilidade do Setor Elétrico (MCSE). Cabe ressaltar que o manual já era utilizado desde 2001.

Alguns estudos já foram realizados quanto ao tema, dentre eles, os de Suzart et al. (2013); Dornelles, Macedo e Rodrigues (2013) e Carvalho, Wanderley, Libonatti e Santos (2014).

Suzart et al. (2013) investigaram as demonstrações contábeis societárias e regulatórias de 62 empresas do setor elétrico, nos anos de 2009 e 2010. Os resultados apresentaram indícios de que, na média, o lucro regulatório foi inferior ao societário, e que as normas societárias adotadas alteraram os retornos com maior intensidade.

O estudo de Dornelles et al. (2013) verificou o conservadorismo das demonstrações contábeis societárias e regulatórias de 82 empresas distribuidoras (concessionárias e permissionárias), nos anos de 2010 e 2011. Os resultados demonstram que a contabilidade regulatória é mais conservadora que a societária, pois, houve uma diminuição do lucro nas demonstrações regulatórias.

Carvalho et al. (2014) analisaram 22 empresas do setor elétrico de 2009 a 2011 e identificaram diferenças significativas nas comparações do grupo de contas patrimoniais e de resultados entre a contabilidade societária e regulatória. Enfim, há diferenças significativas nos resultados das concessionárias quando as demonstrações societárias e regulatórias são comparadas.

\subsection{Estudos Correlatos}

Alguns estudos já foram realizados quanto ao tema, mas não analisaram o setor elétrico especificamente e consequentemente a contabilidade regulatória, esta é a contribuição deste artigo. Kronbauer et al. (2012a) analisaram 36 empresas integrantes do Novo Mercado da BM\&FBOVESPA, de 2006 a 2008, quanto ao reconhecimento dos ativos fiscais diferidos na estrutura financeira e desempenho econômico das mesmas. Constataram que o reconhecimento de AFDs impactam significativamente os índices de liquidez e de endividamento, e ainda que não há 
associação significativa entre os índices de AFD e os demais índices financeiros.

Kronbauer, Souza, Alves e Rojas (2010) investigaram fatores determinantes que podem explicar o nível de reconhecimento de AFD. Encontraram indícios de que os AFDs podem ser utilizados de modo oportunista para melhorar os indicadores financeiros e econômicos das empresas. A amostra foi composta de 29 empresas participantes do IBOVESPA nos anos de 2003 a 2008.

Pereira e Barbosa (2015) analisaram 19 empresas do Setor de Construção Civil de capital aberto quanto aos principais fatos contábeis que motivaram o registro de impostos de diferidos em 2012. Os resultados demonstram que os prejuízos fiscais e a base negativa da contribuição social são os principais motivos para o registro de AFD e os passivos fiscais diferidos foram registrados devido as diferenças entre o lucro tributável e contábil.

Madeira e Costa Júnior (2016) investigaram as motivações econômicas que explicam o nível de reconhecimento dos tributos diferidos sobre o lucro em 68 empresas abertas não financeiras de 2010 a 2013. Os resultados sugerem que as empresas utilizam da discricionariedade proporcionada pela regulação contábil dos tributos diferidos para melhorar a sua rentabilidade.

Kronbauer, Rojas, Ott e Souza (2012b) investigaram a relação que pode existir entre o nível de reconhecimento de impostos diferidos passivos e variáveis explicativas relacionadas com a situação econômica, financeira e tamanho das empresas. A amostra é composta de empresas espanholas e brasileiras durante os anos de 2003 a 2005. Os resultados demostram que a variação das empresas RPFD Ibovespa, explica-se em cerca de 95\%, variando a proporção de Endividamento e nas empresas espanholas IBEX-35, a variação RPFD é explicada para 82\%, da variação de endividamento e do tamanho da empresa.

Rojas, Herrera, Kronbauer e Souza (2010) analisaram os fatores que podem determinar a decisão de ativar os ativos fiscais diferidos em 124 empresas cotadas no período de 1999 a 2001. Os resultados mostram que os níveis de dívida, a liquidez e rentabilidade são determinantes de peso, para o reconhecimento dos ativos fiscais diferidos.

Junqueira e Nakao (2013) buscaram evidências sobre o papel dos ativos e passivos diferidos como instrumento para atingir o nível de capital regulatório pelas empresas financeiras brasileiras de 2004 a 2009. O resultado mostrou que as empresas utilizaram os tributos diferidos para atingir o nível de capital mínimo exigido pelo acordo da Basileia, bem como que o volume de capital regulatório influencia o volume de tributos diferidos.

Esse estudo vem complementar os estudos já existentes, considerando um setor específico que é o setor elétrico e que apresenta dois tipos de demonstrações financeiras a societária e a regulatória. Diante do exposto, apesar de já existirem alguns estudos, há muito ainda a se pesquisar e esse artigo contribui para diminuir esta lacuna.

\section{Metodologia}

A população deste estudo é composta pelas 82 concessionárias de energia elétrica dos três setores: distribuição, geração e transmissão registradas na ANEEL ao final de 2016. Foram coletadas as demonstrações contábeis societárias e regulatórias de cada concessionária de 2014 e 2015. A amostra final, por indisponibilidade de alguns dados ou por não possuir Ativo Fiscal Diferido em seu balanço patrimonial no período analisado, foi composta por 30 concessionárias, sendo 23 distribuidoras, 03 geradoras, 01 transmissora e 03 que são geradoras e transmissoras conforme ilustrado na Figura 1.

Utilizando o modelo de Kronbauer et al. (2012a), os índices foram calculados com os dados extraídos das demonstrações contábeis societárias e regulatórias. Os indicadores escolhidos possuem relação direta com o reconhecimento ou não dos AFDs, ou seja, eles são impactados quando há a contabilização de AFD. 
Figura 1 - Empresas constantes da amostra

\begin{tabular}{|c|c|}
\hline Empresa & Setor \\
\hline Aes Sul Distribuidora Gaúcha S.A & Distribuição \\
\hline Ampla Energia e Serviços S.A & Distribuição \\
\hline Celesc Distribuição S.A & Dis tribuição \\
\hline CELG Geração e Transmis são S.A. & Geração e Transmissão \\
\hline Companhia Energética de Pernambuco - CELPE & Distribuição \\
\hline Cemig Distribuição S.A & Distribuição \\
\hline Companhia Energética de São Paulo - CESP & Geração \\
\hline Companhia Energética do Ceára - COELCE & Distribuição \\
\hline Copel Dis tribuição S.A & Dis tribuição \\
\hline Copel Geração e Distribuição S.A & Geração e Transmissão \\
\hline Companhia Energética do Rio Grande do Norte - Cosern & Distribuição \\
\hline Companhia Jaguari de Energia - CPFL Jaguari & Distribuição \\
\hline Companhia Paulista de Força e Luz - CPFL Paulista & Distribuição \\
\hline Companhia de Transmissão de Energia Elétrica Paulista - CTEEP & Transmissão \\
\hline Empresa de Distribuição de Energia Vale Paranapanema S.A - EDEVP & Distribuição \\
\hline Elektro Eletricidade e Serviços S.A & Distribuição \\
\hline Centrais Elétricas do Norte do Brasil S.A - Eletronorte & Geração e Transmissão \\
\hline Eletrobrás Distribuição Piauí & Distribuição \\
\hline Eletropaulo Metropolitana Eletricidade de São Paulo S.A. & Dis tribuição \\
\hline Energis a Borborema - Distribuidora de Energia S.A & Dis tribuição \\
\hline Energis a Minas Gerais - Distribuidora de Energia S.A & Distribuição \\
\hline Energis a Mato Grosso - Distribuidora de Energia S.A & Dis tribuição \\
\hline Energis a Mato Gross o do Sul - Distribuidora de Energia S.A & Distribuição \\
\hline Energis a Paraíba - Distribuidora de Energia S.A & Dis tribuição \\
\hline Energis a Sergipe - Dis tribuidora de Energia S.A & Dis tribuição \\
\hline Energis a Tocantins - Distribuidora de Energia S.A & Distribuição \\
\hline Espirito Santo Centrais Elétricas S.A - Escelsa & Distribuição \\
\hline Itapebi Geração de Energia S.A & Geração \\
\hline Norte Energia S.A & Geração \\
\hline Rio Grande Energia S.A & Dis tribuição \\
\hline
\end{tabular}

Fonte: Elaborado pela autora

Cada categoria de índices foi calculada para cada empresa, nos dois períodos analisados e com os dados da contabilidade societária e regulatória, de acordo com os seguintes critérios:

(i) LC - Liquidez Corrente: obtida pela divisão do valor do Ativo Circulante pelo valor do Passivo Circulante;

(ii) LG - Liquidez Geral: obtida a partir da divisão entre o valor do Ativo Total e pelo valor do Exigível Total (Circulante e Não Circulante);

(iii) ENDTO - Endividamento obtido pela divisão entre Exigível Total e Ativo Total;

(iv) RPL - Rentabilidade do Patrimônio Líquido obtido a partir da divisão do Lucro Líquido e Patrimônio Líquido Médio (PL inical + PL final/2)

Os índices LG e ENDTO foram calculados com e sem AFD. A partir dos resultados obtidos com os cálculos desses dois índices, com e sem AFD foi utilizada a técnica estatística de diferença de médias para verificar se o reconhecimento de AFDs interfere na situação econômica e financeira das empresas da amostra. 
Os índices LC e RPL foram analisados somente com o AFD, pois, observou-se que as empresas analisadas só possuíam no período analisado AFD de longo prazo, ou seja, no ativo não circulante.

Para definir o teste de média a ser aplicado, realizou-se o teste de normalidade dos dados Kolmogorov-Smirnov (K-S), que é aquele indicado para o tamanho da amostra utilizada. Se a distribuição for normal ao nível de significância acima de 5\% (p>0,05), será utilizado o teste paramétrico t de Student; caso contrário $(\mathrm{p}<0,05)$, o teste a ser utilizado é o de Wilcoxon, que é um teste não paramétrico.

Quanto a variável RPL, no balanço societário, as empresas AES SUL, AMPLA, CESP, Energisa Sergipe e Norte Energia foram retiradas dessa análise em 2015, pois apresentaram prejuízo e a Eletrobrás Piauí porque apresentou prejuízo e patrimônio líquido descoberto. Em 2014, foram retiradas a CELG-GT, Eletropaulo, Norte Energia apresentaram prejuízo e a Eletrobrás Piauí patrimônio líquido negativo.

Para a análise dos dados do balanço regulatório foi retirada a Eletrobrás Piauí por não possuir ativo fiscal diferido, portanto, a amostra final foi de 29. Quanto a variável RPL, as empresas AES SUL, AMPLA, CELESC-D, CELPE, CEMIG-D, Eletrobrás Eletronorte, Energisa Mato Grosso, Energisa Sergipe e Norte Energia foram retiradas dessa análise em 2015, pois apresentaram prejuízo. Em 2014, foram retiradas a CELG-GT, Energisa MS e Norte Energia apresentaram prejuízo.

Foi calculado também o índice de ativos fiscais diferidos (IAFD) que foi obtido pela divisão do AFD pelo Ativo Total. Por fim, realizou-se análise de correlação, visando verificar se o nível de reconhecimento de AFDs possui associação significativa com as variáveis inerentes à situação financeira e econômica das empresas estudadas. Para tanto, foram calculados os coeficientes de correlação de Pearson entre as variáveis analisadas.

\section{Análise dos Resultados}

Após os procedimentos metodológicos, são expostos os resultados encontrados nos demonstrativos societários e regulatórios.

\subsection{Análise dos Resultados dos índices LG e ENDTO com os dados dos balanços societários de 2015 e 2014 com e sem AFD}

Para uma análise geral dos dados foram apuradas a média, mediana, desvio-padrão, assimetria e curtose para os dois anos da amostra.

Tabela 1 - Estatística Descritiva dos índices LG e ENDTO com e sem AFD

\begin{tabular}{c|c|c|r|r|r|r|r}
\hline \multirow{2}{*}{ Indicador } & Ano & Variável & Média & Mediana & Desvio-padrão & Assimetria & Curtose \\
\hline \multirow{4}{*}{ LG } & 2015 & AFD & 1,673148 & 1,474570 & 0,7569 & 2,476 & 7,265 \\
\cline { 2 - 9 } & 2015 & SFD & 1,605123 & 1,396027 & 0,7466 & 2,527 & 7,39 \\
\cline { 2 - 9 } & 2014 & AFD & 1,746516 & 1,594742 & 0,6839 & 2,313 & 6,802 \\
\cline { 2 - 9 } & 2014 & SFD & 1,658195 & 1,474800 & 0,6774 & 2,361 & 6,804 \\
\hline \multirow{5}{*}{ aENDTO } & 2015 & AFD & 0,679520 & 0,678163 & 0,2256 & 0,931 & 3,944 \\
\cline { 2 - 9 } & 2015 & SFD & 0,710384 & 0,716605 & 0,2317 & 0,274 & 2,816 \\
\cline { 2 - 9 } & 2014 & AFD & 0,633125 & 0,62706 & 0,1796 & 0,056 & 0,73 \\
\cline { 2 - 8 } & 2014 & SFD & 0,670040 & 0,678065 & 0,1887 & $-0,22$ & 0,301 \\
\hline
\end{tabular}

Fonte: Dados da Pesquisa 
A Tabela 1, traz as estatísticas descritivas da amostra analisada, com o objetivo de resumilos sem inferir sobre eles. Assim, verificou-se que:

- As variáveis AFD e SFD, em relação ao índice ENDTO, com exceção da variável SFD de 2014 , apresentaram assimetria próximas a zero, indicando que estas variáveis possuem distribuição simétrica.

- O índice ENDTO, em relação a variável SFD, em 2014, apresentou assimetria negativa, indicando uma maior concentração de valores acima da média.

- O índice LG, em ambas as variáveis, nos dois anos analisados, houve a identificação de valores de assimetria positivos e superiores a 1 , indicando uma maior concentração de valores abaixo da média;

- O índice de ENDTO, exceto a variável AFD em 2015, apresentou curtose inferior a três, o que representa que há concentração dos dados ao redor da média;

- O índice LG em todas as variáveis e o índice de ENDTO para a variável AFD em 2015 apresentaram curtose superior a três o que significa que estas possuem uma distribuição mais dispersa;

- Os índices LG e ENDTO nos dois anos e para ambas variáveis apresentam desvio-padrão inferior à média, o que implica em baixa dispersão dos dados analisados.

- Em relação à média, o índice LG melhorou com o reconhecimento de AFD por parte das empresas. Já o índice de ENDTO diminui nos dois anos analisados. Esse resultado vem ao encontro do estudo de Kronbauer et al. (2012a), para estes dois índices.

Diante do exposto, apesar de ser o resultado esperado o importante é saber se a diferença entre as variáveis para os dois índices é estaticamente diferente ou não.

Considerando o tamanho da amostra, foi realizado o teste de Kolmogorov-Smirnov, para verificar se a amostra possui ou não distribuição normal. Na Tabela 2, são apresentados os resultados dos testes.

Tabela 2 - Teste de normalidade Kolmogorov-Smirnov

\begin{tabular}{c|c|c|c|c}
\hline \multirow{2}{*}{ Indicador } & Ano & Variável & Estatística & Probabilidade \\
\hline \multirow{4}{*}{ LG } & 2015 & AFD & 0,215 & 0,001 \\
\cline { 2 - 5 } & 2015 & SFD & 0,225 & 0,000 \\
\cline { 2 - 5 } & 2014 & AFD & 0,237 & 0,001 \\
\cline { 2 - 5 } & 2014 & SFD & 0,221 & 0,000 \\
\hline \multirow{4}{*}{ ENDTO } & 2015 & AFD & 0,122 & $\mathbf{0 , 2 0 0}$ \\
\cline { 2 - 5 } & 2015 & SFD & 0,107 & $\mathbf{0 , 2 0 0}$ \\
\cline { 2 - 5 } & 2014 & AFD & 0,101 & $\mathbf{0 , 2 0 0}$ \\
\cline { 2 - 5 } & 2014 & SFD & 0,084 & $\mathbf{0 , 2 0 0}$ \\
\hline
\end{tabular}

Fonte: Dados da Pesquisa

Segundo os resultados dos testes de Kolmogorov-Smirnov, o índice LG para os dois anos apresentou indícios que as variáveis não possuem distribuição normal, visto que as probabilidades foram inferiores ao nível de significância de 5\% e para o índice de ENDTO, as variáveis possuem indícios de distribuição normal.

Conforme foi explicado no capítulo de metodologia, não possuindo distribuição normal, aplicou-se o teste de Wilcoxon para o índice LG e o teste $t$ de sutdent para o índice ENDTO que apresentou normalidade de dados. 
Tabela 3 - Resultados do teste de médias

\begin{tabular}{|c|c|c|c|c|c|}
\hline \multirow[b]{2}{*}{ Indicador } & \multirow[b]{2}{*}{ Ano } & \multicolumn{2}{|c|}{ Wilcoxon } & \multicolumn{2}{|c|}{ t de Student } \\
\hline & & Estatística & Probabilidade & Estatística & Probabilidade \\
\hline LG & 2015 & $-4,782$ & 0,000 & - & - \\
\hline ENDTO & 2015 & & & $-5,536$ & 0,000 \\
\hline LG & 2014 & $-4,782$ & 0,000 & & \\
\hline ENDTO & 2014 & & & $-6,812$ & 0,000 \\
\hline
\end{tabular}

Fonte: Dados da pesquisa

Os resultados expostos na Tabela 3 demonstram que na média há diferença significativa, quando há reconhecimento ou não da AFD, ou seja, o reconhecimento dos AFDs aumenta a liquidez geral das empresas da amostra em níveis estatisticamente significantes para os dois anos analisados. Já o ENDTO, também há diferença estatística significativa, ou seja, o reconhecimento da AFD diminui o índice de endividamento das empresas para os dois anos analisados.

Os resultados encontrados vêm ao encontro do estudo anterior de Kronbauer et al. (2012a). Portanto, os elaboradores das demonstrações contábeis podem utilizar da discricionariedade do reconhecimento do AFD para 'maquiar' de maneira oportunista esses dois índices financeiros das empresas.

Para complementar o estudo foi calculado o IAFD, LC e RPL conforme comentado na metodologia. Assim, foi realizada uma análise de associação entre o IAFD e os demais índices econômicos, e agora são utilizados os quatros índices LC, LG, ENDTO e RPL. Antes de analisar o resultado da análise de correlação apresenta-se a estatística descritiva dos índices IAFD, LC e RPL, na Tabela 4.

Tabela 4 - Estatística Descritiva dos índices IAFD, LC e RPL

\begin{tabular}{c|c|c|c|c|c|c}
\hline \multirow{2}{*}{ Indicador } & Ano & Média & Mediana & Desvio-padrão & Assimetria & Curtose \\
\hline \multirow{2}{*}{ IAFD } & 2015 & 0,038531 & 0,031380 & 0,0286 & 0,730 & $-0,213$ \\
\cline { 2 - 8 } & 2014 & 0,048477 & 0,046895 & 0,0300 & 0,908 & 1,271 \\
\hline \multirow{2}{*}{ LC } & 2015 & 1,499946 & 1,171239 & 2,1346 & 5,269 & 28,466 \\
\cline { 2 - 8 } & 2014 & 1,647251 & 1,258983 & 1,4794 & 4,126 & 28,466 \\
\hline \multirow{2}{*}{ RPL } & 2015 & 0,151779 & 0,141831 & 0,1076 & 0,918 & 0,587 \\
\cline { 2 - 8 } & 2014 & 0,171691 & 0,157459 & 0,1052 & 1,49 & 3,836 \\
\hline
\end{tabular}

Fonte: Dados da Pesquisa

A Tabela 4, traz as estatísticas descritivas da amostra analisada, com o objetivo de resumilos sem inferir sobre eles. Assim, em relação aos dados, verificou-se que:

- Todos os índices apresentaram assimetria positiva, indicando uma concentração de dados, abaixo da média.

- O IAFD nos dois anos e o índice RPL no ano de 2015 apresentaram curtose inferior a três, o que representa que há concentração dos dados ao redor da média;

- O índice LC nos dois anos e RPL para o ano de 2014 apresentaram curtose superior a três o que significa que estas possuem uma distribuição mais dispersa;

- Todos os índices, com exceção do índice LC no ano de 2015, apresentam desvio-padrão inferior à média, o que implica em baixa dispersão dos dados analisados.

Após a análise descritiva, realizou-se a análise de correlação com cada índice financeiro e econômico, conforme ilustrado na Tabela 5. 
Tabela 5: Correlação de Pearson entre o IAFD e os demais índices

\begin{tabular}{c|c|c|c}
\hline Indicador & Ano & Correlação & Probabilidade \\
\hline LC & 2015 & $-0,253$ & 0,178 \\
\hline LC & 2014 & $-0,351$ & 0,057 \\
\hline LG & 2015 & $-0,067$ & 0,725 \\
\hline LG & 2014 & $-0,158$ & 0,406 \\
\hline RPL & 2015 & $-0,290$ & 0,119 \\
\hline RPL & 2014 & 0,181 & 0,339 \\
\hline ENDTO & 2015 & $-0,116$ & 0,542 \\
\hline ENDTO & 2014 & $-0,002$ & 0,991 \\
\hline
\end{tabular}

Fonte: Dados da Pesquisa

Analisando a Tabela 5, pode-se inferir que não há associação significativa entre o IAFD e os índices financeiros e econômicos. Somente o índice RPL obteve uma correlação positiva mas classificada como fraca, pois é menor que 0,3 . Os demais índices e em todos os anos percebe-se correlação negativa fraca, com exceção da LC em 2014, que possui uma correlação média, com o IAFD. Portanto, não há indícios de que o IAFD das empresas da amostra nos dois anos analisados está relacionado com a melhoria dos índices nas demonstrações financeiras das empresas analisadas. Esse resultado também vem ao encontro dos estudos de Kronbauer et al. (2012a).

Assim, os resultados ratificam os estudos anteriores, ressaltando a relevância na análise do reconhecimento ou não da AFD. Na próxima seção realiza-se as mesmas análises para os balanços regulatórios, em que ainda não há estudos para comparação com os resultados, entretanto, podese avaliar as diferenças entre as contabilidades societária e regulatória.

\subsection{Resultados encontrados com os dados dos balanços regulatórios}

Como realizado no item 4.1 foram apuradas a média, mediana, desvio-padrão, assimetria e curtose para os dois anos da amostra.

Tabela 6 - Estatística Descritiva dos índices LG e ENDTO com e sem AFD

\begin{tabular}{c|c|c|r|r|r|r|r}
\hline \multirow{2}{*}{ Indicador } & Ano & Variável & \multicolumn{1}{c|}{ Média } & \multicolumn{1}{c}{ Mediana } & Desvio-padrão & \multirow{2}{*}{ Assimetria } & \multicolumn{1}{c}{ Curtose } \\
\hline \multirow{4}{*}{ LG } & 2015 & AFD & 1,637664 & 1,397932 & 0,7412 & 2,52 & 6,534 \\
\cline { 2 - 9 } & 2015 & SFD & 1,576119 & 1,345194 & 0,7272 & 2,51 & 6,322 \\
\cline { 2 - 8 } & 2014 & AFD & 1,695451 & 1,488064 & 0,7151 & 2,044 & 5,095 \\
\cline { 2 - 9 } & 2014 & SFD & 1,620286 & 1,410240 & 0,7056 & 2,031 & 4,839 \\
\hline \multirow{4}{*}{ ENDTO } & 2015 & AFD & 0,684374 & 0,715342 & 0,1839 & $-0,884$ & 0,477 \\
\cline { 2 - 8 } & 2015 & SFD & 0,644948 & 0,682560 & 0,182 & $-0,78$ & 0,276 \\
\cline { 2 - 8 } & 2014 & AFD & 0,666196 & 0,67203 & 0,2143 & 0,484 & 1,803 \\
\cline { 2 - 8 } & 2014 & SFD & 0,618660 & 0,615411 & 0,2103 & 0,676 & 2,269 \\
\hline
\end{tabular}

Fonte: Dados da Pesquisa

A Tabela 6, traz as estatísticas descritivas da amostra analisada, com o objetivo de resumilos sem inferir sobre eles. Assim, em relação aos dados regulatórios, verificou-se que:

- As variáveis AFD e SFD, em relação ao índice ENDTO, em 2014, apresentaram assimetria próximas a zero, indicando que estas variáveis possuem distribuição simétrica. Já em 2015, apresentou assimetria negativa, indicando uma maior concentração de valores acima da 
média;

- O índice de ENDTO, apresentou curtose inferior a três, o que representa que há concentração dos dados ao redor da média;

- O índice LG apresentou curtose superior a três o que significa que estas possuem uma distribuição mais dispersa;

- Os índices LG e ENDTO nos dois anos e para ambas variáveis apresentam desvio-padrão inferior à média, o que implica em baixa dispersão dos dados analisados.

- Em relação à média, o índice de LG melhorou com o reconhecimento de AFD por parte das empresas. Já o índice de ENDTO aumenta nos dois anos analisados. O resultado do índice LG coincide com a análise com os dados do balanço societário. Entretanto, quanto ao ENDTO ocorreu o oposto ao encontrado nos dados societários, em que o índice de ENDTO diminui. Portanto, o reconhecimento de AFD, no índice de ENDTO na contabilidade regulatória não contribui para a melhora dos resultados. Analisando os dados esse resultado pode ser explicado porque o passivo exigível regulatório é maior nos dois anos analisados em mais de $15 \%$ em relação ao passivo exigível societário.

Diante do exposto, em relação ao índice de ENDTO o resultado não era esperado, resta saber se a diferença entre as variáveis para os dois índices é estaticamente diferente ou não.

Igualmente a análise dos dados societários, realizou-se o teste de normalidade KS, os resultados estão expostos na Tabela 7.

Tabela 7: Teste de normalidade Kolmogorov-Smirnov

\begin{tabular}{c|c|c|c|c}
\hline \multirow{2}{*}{ Indicador } & Ano & Variável & Estatística & Probabilidade \\
\hline \multirow{4}{*}{ LG } & 2015 & AFD & 0,247 & 0,000 \\
\cline { 2 - 5 } & 2015 & SFD & 0,241 & 0,000 \\
\cline { 2 - 5 } & 2014 & AFD & 0,237 & 0,000 \\
\cline { 2 - 5 } & 2014 & SFD & 0,264 & 0,000 \\
\hline \multirow{4}{*}{ ENDTO } & 2015 & AFD & 0,116 & $\mathbf{0 , 2 0 0}$ \\
\cline { 2 - 5 } & 2015 & SFD & 0,116 & $\mathbf{0 , 2 0 0}$ \\
\cline { 2 - 5 } & 2014 & AFD & 0,114 & $\mathbf{0 , 2 0 0}$ \\
\cline { 2 - 5 } & 2014 & SFD & 0,091 & $\mathbf{0 , 2 0 0}$ \\
\hline
\end{tabular}

Fonte: Dados da Pesquisa

Segundo os resultados dos testes de Kolmogorov-Smirnov, o índice LG para os dois anos apresentou indícios que as variáveis não possuem distribuição normal, visto que as probabilidades foram inferiores ao nível de significância de 5\% e para o índice de ENDTO, as variáveis possuem indícios de distribuição normal.

Mesmo resultado encontrado na análise com os dados societários e como já dito, aplicou-se o teste de Wilcoxon para o índice LG e o teste $t$ de sutdent para o índice ENDTO que apresentou normalidade de dados.

Os resultados evidenciados na Tabela 8 demonstram que na média há diferença significativa, quando há reconhecimento ou não da AFD, ou seja, o reconhecimento dos AFDs aumenta a liquidez geral das empresas da amostra em níveis estatisticamente significantes para os dois anos analisados. Já o ENDTO, também há diferença estatística significativa, ou seja, o reconhecimento da AFD aumenta o índice de endividamento das empresas para os dois anos analisados 
Tabela 8: Resultados do teste de médias

\begin{tabular}{c|r|r|r|r|r}
\hline \multicolumn{2}{c|}{} & \multicolumn{2}{|c|}{ Wilcoxon } & \multicolumn{2}{c}{ t de Student } \\
\hline Indicador & Ano & \multicolumn{1}{c|}{ Estatística } & Probabilidade & Estatística & Probabilidade \\
\hline LG & 2015 & $-4,703$ & 0,000 & - & - \\
\hline ENDTO & 2015 & & & 9,263 & 0,000 \\
\hline LG & 2014 & $-4,782$ & 0,000 & & 0,000 \\
\hline ENDTO & 2014 & & & 9,302 & \\
\hline
\end{tabular}

Fonte: Dados da pesquisa

Mais uma vez, destaca-se que houve diferença nos resultados entre os dados societários e regulatórios quanto ao índice de ENDTO, enquanto no balanço societário, o índice do endividamento diminui quando há o reconhecimento de AFD nos balanços regulatórios é o oposto esse índice aumenta, portanto, não melhora os resultados da empresa.

Tabela 9: Estatística Descritiva dos índices IAFD, LC e RPL

\begin{tabular}{c|c|c|c|c|c|c}
\hline \multirow{2}{*}{ Indicador } & Ano & Média & Mediana & Desvio-padrão & Assimetria & Curtose \\
\hline \multirow{2}{*}{ IAFD } & 2015 & 0,039426 & 0,040199 & 0,022919 & 0,184 & $-0,324$ \\
\cline { 2 - 7 } & 2014 & 0,047535 & 0,04563 & 0,027525 & 0,717 & 1,039 \\
\hline \multirow{2}{*}{ LC } & 2015 & 1,374319 & 1,120263 & 1,4608 & 5,058 & 26,651 \\
\cline { 2 - 8 } & 2014 & 1,478972 & 1,179180 & 1,1853 & 4,051 & 18,57 \\
\hline \multirow{2}{*}{ RPL } & 2015 & 0,617677 & 0,056941 & 0,0554 & 1,44 & 2,013 \\
\cline { 2 - 8 } & 2014 & 0,136117 & 0,143472 & 0,8374 & $-0,083$ & $-0,805$ \\
\hline
\end{tabular}

Fonte: Dados da Pesquisa

A Tabela 9, traz as estatísticas descritivas da amostra analisada, com o objetivo de resumilos sem inferir sobre eles. Assim, verificou-se que:

- Todos índices apresentaram assimetria positiva, indicando uma concentração de dados, abaixo da média, com exceção do índice RPL no ano de 2014;

- O IAFD e RPL nos dois anos apresentaram curtose inferior a três, o que representa que há concentração dos dados ao redor da média;

- O índice LC nos dois anos apresentou curtose superior a três o que significa que estas possuem uma distribuição mais dispersa;

- Todos os índices, com exceção do índice LC no ano de 2015 e RPL ano de 2014, apresentam desvio-padrão inferior à média, o que implica em baixa dispersão dos dados analisados.

Após a análise descritiva, realizou-se a análise de correlação com cada índice financeiro e econômico, conforme ilustrado na Tabela 10. Analisando a Tabela 10, pode-se inferir que não há associação significativa entre o IAFD e os índices financeiros e econômicos. Mesmo resultado encontrado com os dados societários.

O índice ENDTO nos dois períodos e o índice RPL em 2014 obtiveram uma correlação positiva, mas classificada como fraca, pois são menores que 0,3 . Os demais índices e em todos os anos percebe-se correlação negativa fraca com o IAFD. Portanto, não há indícios de que o IAFD das empresas da amostra nos dois anos analisados está relacionado com a melhoria dos índices nas demonstrações regulatórias das empresas analisadas. Mesmo resultado encontrado nas demonstrações societárias. 
Tabela 10: Correlação de Pearson entre o IAFD e os demais índices

\begin{tabular}{c|c|c|c}
\hline Indicador & Ano & Correlação & Probabilidade \\
\hline LC & 2015 & $-0,346$ & $\mathbf{0 , 6 6 0}$ \\
\hline LC & 2014 & $-0,28$ & $\mathbf{0 , 1 4 1}$ \\
\hline LG & 2015 & $-0,184$ & $\mathbf{0 , 3 3 6}$ \\
\hline LG & 2014 & $-0,286$ & $\mathbf{0 , 1 3 3}$ \\
\hline RPL & 2015 & $-0,160$ & $\mathbf{0 , 4 0 7}$ \\
\hline RPL & 2014 & $\mathbf{0 , 1 1 5}$ & $\mathbf{0 , 5 5 1}$ \\
\hline ENDTO & 2015 & $\mathbf{0 , 1 4 3}$ & $\mathbf{0 , 4 5 8}$ \\
\hline ENDTO & 2014 & $\mathbf{0 , 2 0 6}$ & $\mathbf{0 , 2 8 3}$ \\
\hline
\end{tabular}

Fonte: Dados da Pesquisa

A próxima seção analisa as diferenças entre a contabilidade societária e regulatória, um dos objetivos secundários do artigo.

\subsection{Análise da Contabilidade Societária x Contabilidade Regulatória}

Para essa análise foram consideradas 29 empresas que possuíam os dados regulatórios, sendo retirada a Eletropaulo Piauí.

Tabela 11 - Média do Lucro Societário x Médio Lucro Regulatório

\begin{tabular}{clcc}
\hline Ano & LLS & LLR & Variação \\
\hline 2015 & 151.396 & 63.189 & $\mathbf{1 4 0 \%}$ \\
2014 & 260.369 & 264.302 & $\mathbf{- 1 \%}$ \\
\hline
\end{tabular}

Fonte: Dados da Pesquisa

Percebe-se uma grande diferença entre o Lucro Líquido Societário - LLS e o Lucro Líquido Regulatório - LLR em 2015, das 29 empresas analisadas. A AES SUL, por exemplo, apresentou um prejuízo de $\$ 4.922 .000$ no balanço societário passando para um prejuízo de $\$ 69.531 .000$. A Celesc-D auferiu um lucro societário de $\$ 81.346 .000$ para um prejuízo regulatório de $\$ 178.112$. A Copel-D divulgou um LLS \$206.054.000 para um LLR \$7.776.000. Portanto, são bastante expressivas as diferenças no lucro no ano de 2015. Já em 2014 quase não houve diferença, sendo o LLR maior que o LLS em 1\%. O resultado de 2015 vem ao encontro dos estudos de Suzart et al. (2013). Segundo Dornelles, Macedo e Rodrigues (2013) as demonstrações regulatórias são mais conservadoras que as societárias, em virtude do lucro reduzido ou aumento do prejuízo nas demonstrações regulatórias.

Tabela 12 - Média do Ativo Total Societário x Ativo Total Regulatório

\begin{tabular}{cccc}
\hline Ano & ATS & ATR & Variação \\
\hline 2015 & 6.826 .784 & 7.681 .207 & $\mathbf{- 1 1 \%}$ \\
2014 & 5.853 .780 & 6.552 .858 & $\mathbf{- 1 1 \%}$ \\
\hline
\end{tabular}

Fonte: Dados da Pesquisa

Analisando a Tabela 12, o Ativo Total Societário - ATS foi menor em 11\% nos dois anos analisados. Tal fato pode ser explicado pelas diferenças nas normas nas classificações dos ativos, como por exemplo, o reconhecimento de ativos regulatórios e também tendo em vista que o ICPC01 os ativos passaram a ser contabilizados como Ativo Financeiro e Intangível de acordo com o contrato de concessão e não mais o reconhecimento como imobilizado, como ainda é 
reconhecido na contabilidade regulatória. Esse resultado corrobora com o estudo de Carvalho et al. (2014).

A Tabela 13 traz os dados do patrimônio líquido em que a diferença entre as duas contabilidades é mínima. Os mesmos resultados foram encontrados por Suzart et al. (2013) e Carvalho et al. (2014).

Tabela 13 - Média do PL Total Societário x PL Total Regulatório

\begin{tabular}{llrrr}
\hline Ano & PLS & PLR & Variação \\
\hline & 2015 & 2.377 .774 & 2.327 .445 & $\mathbf{2 \%}$ \\
& 2014 & 2.289 .872 & 2.308 .041 & $\mathbf{- 1 \%}$ \\
\hline
\end{tabular}

Fonte: Dados da Pesquisa

Enfim, apesar de não ser o foco do artigo, é interessante e relevante acompanhar as diferenças entre a contabilidade regulatória e societária. As diferenças podem ser explicadas tendo em vista o objetivo de cada uma.

É o que Suzart et al. (2013) destaca: enquanto as normas societárias buscam informações mais relevantes para os investidores, as normas regulatórias visam tornar mais transparente o processo de formação da tarifa.

\section{Considerações Finais}

Este artigo teve por objetivo geral analisar se o reconhecimento de AFDs interfere na situação financeira das empresas do setor elétrico brasileiro, tanto nas demonstrações societárias como nas demonstrações regulatórias.

Com uma amostra de 30 empresas do setor elétrico, foi demonstrado que há diferença significativa entre os índices LG e ENDTO quando do reconhecimento ou não do AFD, nos dados societários e regulatórios. Entretanto, nos dados societários ambos os índices melhoram o desempenho financeiro da empresa quando há o reconhecimento do AFD. Com os dados regulatórios essa melhora ocorre somente com o índice LG. Quanto ao ENDTO o efeito é contrário, o reconhecimento do AFD aumenta o índice de ENDTO.

Esse resultado vem ao encontro de estudos anteriores e assim devido o montante a ser contabilizado pode variar segundo o perfil dos gestores e podem ocorrer comportamentos oportunistas com o objetivo de 'maquiar' os índices financeiros das empresas. Outro ponto seria a falta comparabilidade das demonstrações contábeis do mesmo setor devido as escolhas contábeis que a norma proporciona.

Em relação ao IAFD, não foram encontradas evidências que o mesmo influencia na melhoria dos índices financeiros e econômicos tanto com os dados societários quanto aos dados regulatórios.

Outra proposta do artigo foi discutir as diferenças entre a contabilidade societária e regulatória. Observou-se que o lucro no ano de 2015 apresentou grande diferença na comparação, o LLR foi menor em $140 \%$. Já o ativo total foi maior em $11 \%$. Essa análise foi realizada para fomentar a discussão sobre o tema. É importante destacar essas diferenças com o intuito do regulador poder rever o custo benefício da contabilidade regulatória.

Pesquisas futuras poderão ser realizadas em outros setores específicos com o objetivo de analisar se os resultados serão os mesmos.

\section{Referências}

CARVALHO, E. S.; WANDERLEY, C. A.; LIBONATTI, J. J.; SANTOS, A. H. S. Um estudo comparativo entre os indicadores econômico-financeiros, baseados na contabilidade regulatória e nas normas contábeis internacionais das empresas de distribuição de energia do setor elétrico 
brasileiro. Registro Contábil - Recont, v. 5, n. 3, p. 20-40, 2014.

DORNELLES, O. M.; MACEDO, M. Á.; RODRIGUES, A. O Conservadorismo Contábil nas Demonstrações Contábeis Financeiras Societárias e Regulatórias das Empresas Distribuidoras de Energia Elétrica. VII Congresso Anpcont. Fortaleza, CE, 2013. Anais..., 2013.

HERBOHN K.; TUTTICCI I.; KHOR, P.S. Changes in Unrecognised Deferred Tax Accruals from Carry-Forward Losses: Earnings Management or Signalling. Journal of Business Finance \& Accounting, 37(7-8), 763-791, 2010. https://doi.org/10.1111/j.1468-5957.2010.02207.x

MARTINS, E.; GELBCKE, E. R.; SANTOS, A.; IUDÍCIBUS, S. Manual de Contabilidade societária: aplicável a todas as sociedades. São Paulo: Atlas, 2013.

JUNQUEIRA, M. A. D. R; NAKAO, S. H. O Papel de Tributos Diferidos no Capital Regulatório de Instituições Financeiras Brasileiras. Revista de Contabilidade e Finanças, v.24, n. 63, p. 195205, 2013. https://doi.org/10.1590/S1519-70772013000300003

KRONBAUER, C. A.; SOUZA, M .A.; ALVES, T. W.; ROJAS, J. M. Fatores determinantes do reconhecimento de ativos fiscais diferidos. Revista Universo Contábil, v. 6, n. 4, p. 68-88, 2010.https://doi.org/10.4270/ruc.2010432

KRONBAUER, C. A.; SOUZA, M. A.; WEBBER, F. L.; OTT, E. R. Impacto do Reconhecimento de Ativos Fiscais Diferidos na Estrutura Financeira e no Desempenho Econômico de Empresas Brasileiras. Revista Contemporânea de Contabilidade, v. 9, n. 17, p. 39-60, 2012 a. http://dx.doi.org/10.5007/2175-8069.2012v9n17p39

KRONBAUER, C. A.; ROJAS, M. J.; OTT, E. R.; SOUZA, M. A. Evidencia de utilización oportunista de normas contables em el reconocimiento de pasivos fiscales diferidos em empresas brasileñas y españolas. Revista de Contabilidade e Organizações, v. 6, n. 16. p. 39-51, 2012 b. https://doi.org/10.11606/rco.v6i16.52666.

MADEIRA, F. L; COSTA JUNIOR, J. V. Características dos Tributos Diferidos nas Companhias Abertas Brasileiras após a adoção das IFRS. Revista de Contabilidade e Controladoria, Curitiba, v. 8, n.3, p. 126-147, 2016. https://doi.org/10.5380/rcc.v8i3.44228.

PAULO, E; MARTINS, E; CORRAR, L. J. Detecção do Gerenciamento de Resultados pela análise do diferimento tributário. Revista de Administração de Empresas, $R A E$, v. 47, n. 1, p. 46-49, 2007.

PEREIRA, T. R. L.; BARBOSA, M. B. Fatores determinantes na evidenciação de impostos diferidos pelas empresas do setor de construção civil listadas na BM\&FBovespa. Revista Mineira de Contabilidade, v. 16, n.12, p. 41-50, 2015.

PEREZ-JUNIOR, J. H.; OLIVEIRA, L. M. (2010). Contabilidade Avançada. 7ed. São Paulo: Editora Atlas, 2010.

ROJAS, M. J.; HERRERA, L. D.; KRONBAUER, C.A.; SOUZA, M.A. La activación de las diferencias temporales positivas en empresas cotizadas españolas: un estudio empírico. Revista Contabilidade, Gestão e Governança, v. 13, n. 1, p. 3-15, 2010. 
REZENDE, G. P.; NAKAO, S. H. Gerenciamento de Resultados e a Relação com o Lucro Tributável das Empresas Brasileiras de Capital Aberto. Revista Universo Contábil. v. 8, n. 1, p. 06-21, jan./mar., 2012. https://doi.org/10.4270/ruc.2012101.

SUZART, J. A.; SOUZA, V. R.; CARVALHO, A. S.; RIVA, P. D.; MARTINS, E.; SALOTTI, B. M. Corporate Information versus Regulatory Information: Relevance of Accounting Information in the Brazilian Electric Sector. International Journal of Business and Social Science, v. 4, n.11, p. 161-175, 2013.

WASSERMAN, C. O ativo fiscal diferido no sistema financeiro nacional: análise e proposta de contabilização. Dissertação de Mestrado, Faculdade de Economia, Administração e Contabilidade, Universidade de São Paulo, São Paulo, SP. Brasil, 2004.

* Uma versão preprint do artigo foi apresentada no XVII USP International Conference in Accounting, em julho de 2017. 